\title{
Cosmic-ray driven dynamo in galaxies
}

\author{
M. Hanasz ${ }^{1}$, D. Wóltanski ${ }^{1}$, K. Kowalik ${ }^{1}$ and H. Kotarba ${ }^{2}$ \\ ${ }^{1}$ Centre for Astronomy, Nicolaus Copernicus University, ul. Gagarina 11, PL-87-100 Torun, \\ Poland \\ ${ }^{2}$ University Observatory Munich, Scheinerstr. 1, D-81679 Munich, Germany
}

\begin{abstract}
We present recent developments of global galactic-scale numerical models of the Cosmic Ray (CR) driven dynamo, which was originally proposed by Parker (1992). We conduct a series of direct $\mathrm{CR}+\mathrm{MHD}$ numerical simulations of the dynamics of the interstellar medium (ISM), composed of gas, magnetic fields and CR components. We take into account CRs accelerated in randomly distributed supernova (SN) remnants, and assume that SNe deposit smallscale, randomly oriented, dipolar magnetic fields into the ISM. The amplification timescale of the large-scale magnetic field resulting from the CR-driven dynamo is comparable to the galactic rotation period. The process efficiently converts small-scale magnetic fields of SN-remnants into galactic-scale magnetic fields. The resulting magnetic field structure resembles the $\mathrm{X}$-shaped magnetic fields observed in edge-on galaxies.
\end{abstract}

Keywords. Galaxies: ISM, Magnetic Fields; ISM: Cosmic Rays, Magnetic Fields; MHD: Dynamos

\section{Introduction}

The dynamical role of CRs was first recognized by Parker, (1966), who noticed that a vertically stratified ISM which consists of thermal gas, magnetic fields and CRs is unstable due to buoyancy of the weightless components, i.e. the magnetic fields and the CRs. According to diffusive shock acceleration models CRs are continuously supplied to the ISM by SN remnants. Therefore, the buoyancy effects caused by CRs are expected in all star forming galaxies. Theories of diffusive shock acceleration predict that about $10 \%$ of the $\sim 10^{51}$ erg of the SN II explosion energy is converted to CR energy. Observational data indicate that gas, magnetic fields and CRs appear in approximate energetic equipartition, which means that all three components are dynamically coupled. In order to incorporate the CR propagation in MHD considerations we use the diffusion-advection equation (e.g. Schlickeiser \& Lerche, 1985) and take into account the CR pressure gradient in the gas equation of motion (see e.g. Berezinski et al., 1990).

The CR-driven dynamo was originally proposed by Parker, 1992. Our model of the CRdriven dynamo involves the following elements (Hanasz et al., 2004; Hanasz et al., 2006; Hanasz et al., 2009b; Hanasz et al., 2009c): (1) The CR nuclear component described by the diffusion-advection transport equation, supplemented to the standard set of resistive MHD equations (Hanasz \& Lesch, 2003). (2) CRs supplied in SN remnants. The CR input of individual SNe is assumed to be $10 \%$ of the typical SN kinetic energy output (= $10^{51} \mathrm{erg}$ ), while the thermal energy output from supernovae is neglected. (3) Anisotropic CR diffusion along magnetic field lines (Giacalone \& Jokipii, 1999), (4) Finite resistivity of the ISM in order to permit topological evolution of the galactic magnetic fields via anomalous resistivity processes (Hanasz et al., 2002), and/or via turbulent reconnection (Kowal et al., 2009) on small spatial scales, which are unresolved in our simulations. (5) An initial gas distribution in the disk which follows the model of the ISM in the Milky 

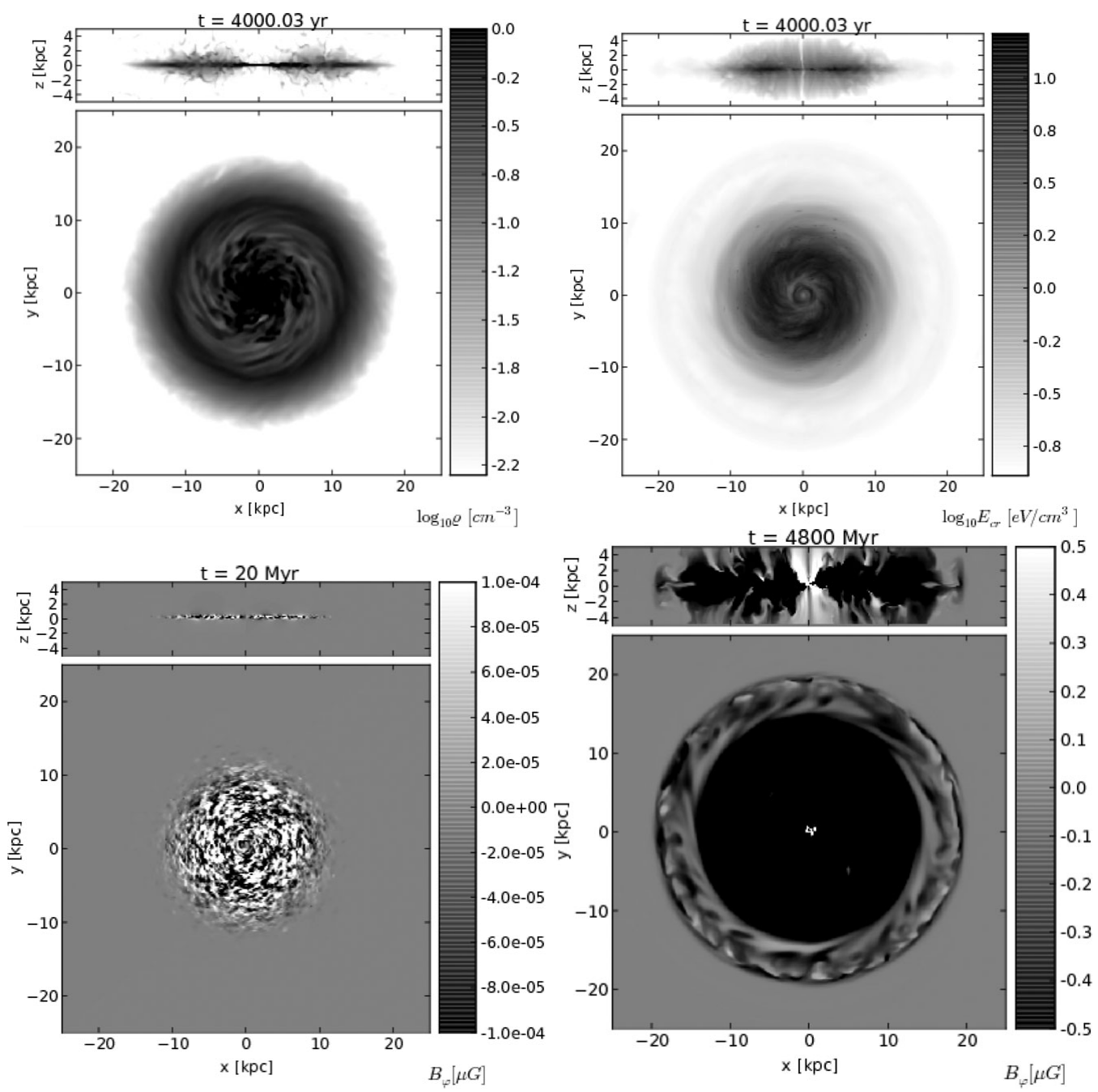

Figure 1. Top panels: logarithm of gas number density (left) and cosmic ray energy density (right) at $t=4 \mathrm{Gyr}$. Bottom panels: the distribution of toroidal magnetic field at $t=20 \mathrm{Myr}$ (left) and $t=4.8 \mathrm{Gyr}$ (right). Unmagnetized regions of the volume are grey, while positive and negative toroidal magnetic fields are marked lighter and darker, respectively. Note that the grey-scale scale in magnetic field maps is saturated to enhance weaker magnetic field structures in disk peripheries.

Way by Ferrière (1998). (6) Differential rotation of the interstellar gas, which currently follows an assumed form of a galactic gravitational potential.

We briefly mention that various properties of the shearing-box models of the CR-driven dynamo were discussed in a series of papers. Computations of the dynamo coefficients in Parker unstable disks with CRs and shear are described by Kowal et al., (2006) and by Otmianowska-Mazur et al., (2007). Synthetic radio-maps of a global galactic disk based on local CR-driven dynamo models exhibiting X-type structures were presented by Otmianowska-Mazur et al.(2009). More recently, Siejkowski et al., (2010) (see also Siejkowski et al., 2010, this volume) demonstrated that the CR-driven dynamo can also work given the physical conditions of irregular galaxies, characterized by a relatively weak rotation and shearing rate. 

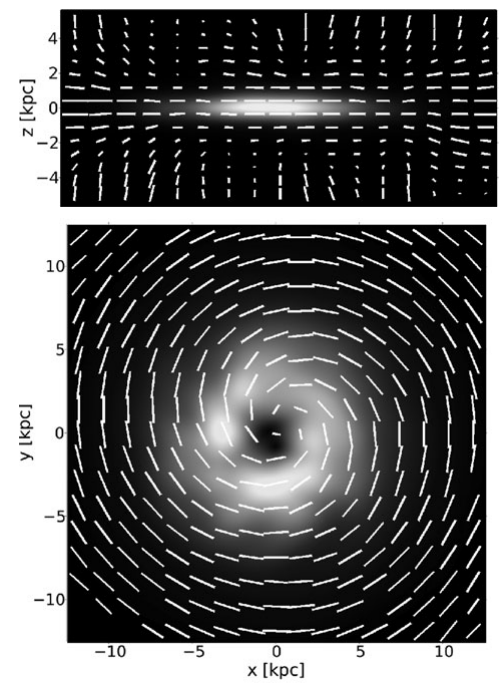

Figure 2. Synthetic radio maps of polarized intensity (PI) of synchrotron emission, together with polarization vectors are shown for the edge-on and face-on views of the galaxy at $t=4.8 \mathrm{Myr}$. Vectors direction resembles electric vectors rotated by $90^{\circ}$, and their lengths are proportional to the degree of polarization.

\section{Global CR-driven dynamo simulations}

Our first realization of a global CR+MHD galactic disk model relies on the following assumptions: First of all, we adopt analytical formulae for the gravitational potential corresponding to a system consisting of a galactic halo, bulge and disk Allen \& Santillan, 1991. We assume no magnetic field at $t=0$, and that weak $\left(10^{-4} \mu \mathrm{G}\right)$, dipolar, small scale $(r \sim 50 \mathrm{pc})$, randomly oriented magnetic fields are supplied locally in $10 \%$ of the SN remnants for $t \leqslant 1$ Gyr. We assume also that the SN rate is proportional to the star formation rate (SFR) which, on the other hand, is proportional to the initial gas column density.

We use the PIERNIK MHD code (Hanasz et al., 2010a; Hanasz et al., 2010b; Hanasz et al., 2008; Hanasz et al., 2009a) which is a grid-MHD code based on the Relaxing TVD (RTVD) scheme (Jin \& Xin, 1995; Pen et al., 2003). PIERNIK is parallelized by means of block decomposition with the aid of the MPI library. The original scheme was extended to deal with dynamically independent but interacting fluids, i.e. thermal gas and a diffusive CR gas, which is described within the fluid approximation (Hanasz et al., 2008).

We find magnetic field amplification originating from the small-scale, randomly oriented dipolar magnetic fields, which is apparent through the exponential growth by several orders of magnitude of both the magnetic flux and the magnetic energy (details see Hanasz et al., 2009). The growth phase of the magnetic field starts at the beginning of the simulation. The growth of the magnetic field strength saturates at about $t=4 \mathrm{Gyr}$, reaching values of $3-5 \mu \mathrm{G}$ in the disk. During the amplification phase, magnetic flux and total magnetic energy grow by about 6 and 10 orders of magnitude, respectively. The average e-folding time of magnetic flux amplification is approximately equal to $270 \mathrm{Myr}$, corresponding to the rotation at the galactocentric radius $(\approx 10 \mathrm{kpc})$. The magnetic field is initially entirely random $(t=20 \mathrm{Myr})$, since it originates from randomly oriented magnetic dipoles. Later on, the toroidal magnetic field component forms a spiral structure revealing reversals in the plane of the disk. The magnetic field structure evolves gradually towards larger and lager scales. The toroidal magnetic field component becomes 

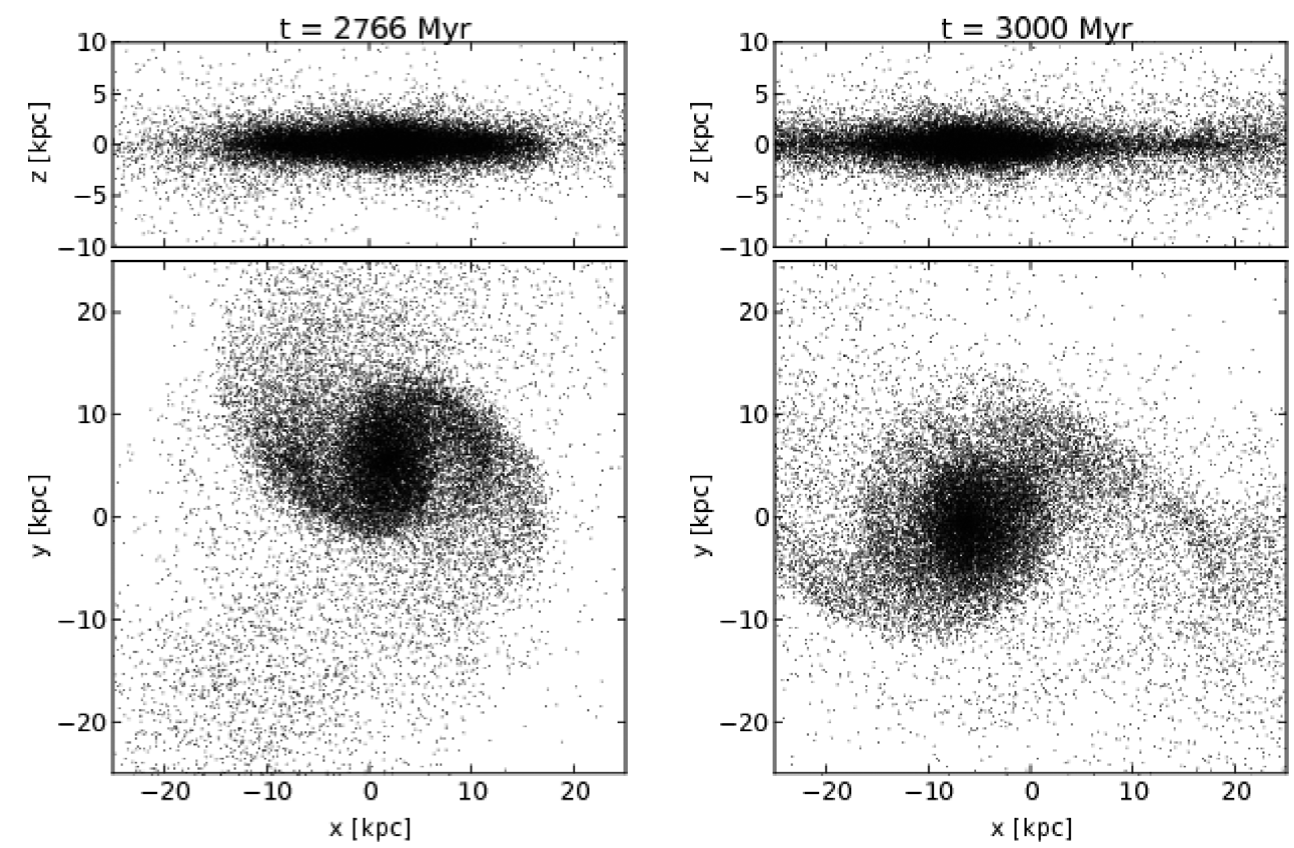

Figure 3. Two states of the N-body galactic disk prior to the galactic merger. The companion galaxy is apparent in the right panels.

almost uniform inside the disk at $t=2.5 \mathrm{Gyr}$. The volume occupied by the well-ordered magnetic field expands continuously until the end of the simulation.

In order to visualize the magnetic field structure in a manner resembling radio observations of external galaxies, we construct synthetic radio maps of the synchrotron radio-emission, assuming that energy density of CR electrons equals $1 \%$ energy density of CR nucleons. We apply standard procedures of line-of-sight integration of the stokes parameters $I, Q$, and $U$ for the polarized synchrotron emissivity. We neglect the effects of of Faraday rotation.

In Figure 2, we show the polarized intensity of synchrotron emission (grey-scale maps), together with polarization vectors. Electric vectors, computed on the basis of integrated Stokes parameters, are rotated by $90^{\circ}$ to reproduce the magnetic field direction averaged along the line-of-sight, assuming vanishing Faraday rotation effects. The polarization vectors, indicating the mean magnetic field direction, reveal a regular spiral structure in the face-on view, and the so-called $X$-shaped structure in the edge-on view. A particular similarity can be noticed between our edge-on synthetic radio map and the radio maps of observed edge-on galaxies such as NGC 891 (Krause, 2009). In the present global model, the X-shaped configuration is an intrinsic property of the magnetic field structure, since it corresponds closely to the flaring radial distribution of magnetic field in the disk and its neighborhood, as shown in Figure 1.

The face-on synchrotron radio map reveals a spiral structure of the magnetic field, however, due to the assumed axisymmetric gravitational potential no features resembling spiral magnetic arms are present. To make the model more realistic, we incorporate non-axisymmetry in the gravitational potential, applying two different approaches. The first approach relies on the addition of an analytical elliptical perturbation to the axisymmetric gravitational potential. The results of this approach, which are presented by Kulpa-Dybel et al., (2010, this volume), indicate that the CR-driven dynamo model 

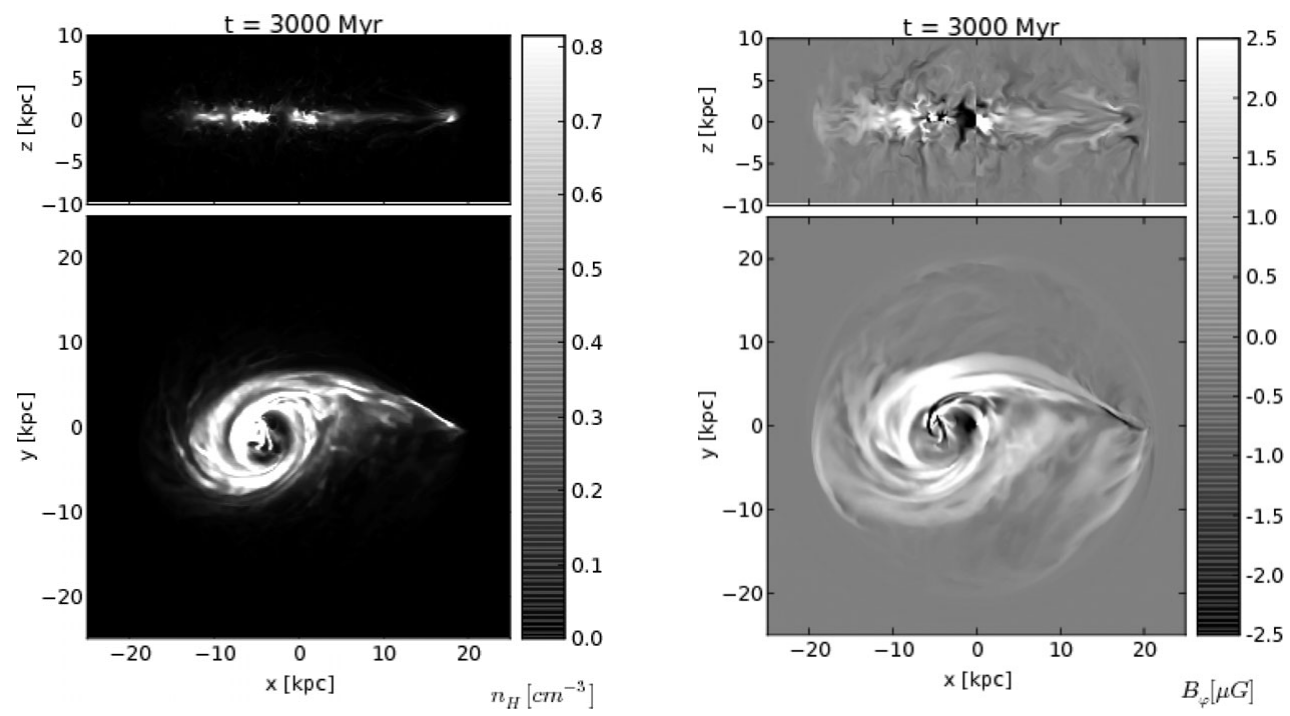

Figure 4. Gas density (left panel) and magnetic field (right panel) corresponding to the gravitational potential of the N-body system at the right plot of Fig.3

reveals new properties, such as the presence of a ring-like structure as well as a shift of the magnetic arms with respect to the crests of spiral density waves.

As a further step towards more realistic galactic magnetic field models we perform N-body simulations of a disk-bulge-halo system (Wóltański et al., 2011), and interpolate the resulting gravitational potential onto the computational grid. We use this potential to compute the gravitational acceleration acting on the fluid components. In order to excite density waves in the galactic disk we add a small satellite galaxy, which ultimately merges with the main galaxy at $t=3.2 \mathrm{Gyr}$. Two snapshots of the $\mathrm{N}$-body disk simulation are displayed in Fig. 3. The N-body part of the computation is performed with the VINE code (Wetzstein et al., 2009), and the CR+MHD part with the PIERNIK code. Fig. 4 shows the gas density and the toroidal magnetic field component. Similar to the case of a axisymmetric gravitational potential we observe efficient magnetic field amplification by the CR-driven dynamo in the presence of density waves in the galactic disk. In the presence of spiral arms in the stellar and gas components the magnetic field also reveals a spiral structure. Moreover, we notice that both polarities of the azimuthal magnetic field are present at an advanced stage of magnetic field evolution. It appears that during the merger phase and afterwards the magnetic field structure becomes even more disordered, providing a possible explanation for the less regular magnetic field structures observed in interacting galaxies such as M51 (Fletcher et al., 2010).

\section{Conclusions}

We have shown that the contribution of CRs to the dynamics of the ISM on a global galactic scale, studied by means of $\mathrm{CR}+\mathrm{MHD}$ simulations, leads to a very efficient magnetic field amplification on the timescale of galactic rotation. The model applying a fixed analytical gravitational potential reveals a large scale regular magnetic field with apparent spiral structure in the face-on view and a X-shaped structure in the edge-on view. In the presence of spiral perturbations excited in the stellar component, the magnetic field structure follows these perturbations in the stellar and gaseous components. The magnetic field structure becomes less regular compared to the axisymmetric case. Dynamical 
magnetic field structures with opposite polarities develop within the disk and are present even at the saturation phase of the dynamo. Moreover, during the coalescence phase of the two galaxies the magnetic field structure becomes irregular as observed in M51. An important part of the CR-driven dynamo is the galactic wind which reaches velocities of a few hundred $\mathrm{km} / \mathrm{s}$ at galactic altitudes of a few kpc. The mass of gas transported out of the disk is about $1 M_{\odot} /$ yr for the star formation rate of the Milky Way.

\section{Acknowledgements}

This work was supported by Polish Ministry of Science and Higher Education through the grants 92/N-ASTROSIM/2008/0 and N N203 511038.

\section{References}

Allen, C. \& Santillan, A.: 1991, Revista Mexicana de Astronomia y Astrofisica 22, 255

Berezinskii, V. S., Bulanov, S. V., Dogiel, V. A., \& Ptuskin, V. S.: 1990, Astrophysics of cosmic rays, Amsterdam: North-Holland, 1990, edited by Ginzburg, V.L.

Ferriere, K.: 1998, ApJ 497, 759

Fletcher, A., Beck, R., Shukurov, A., Berkhuijsen, E. M., \& Horellou, C.: 2010, ArXiv e-prints Giacalone, J. \& Jokipii, J. R.: 1999, ApJ 520, 204

Hanasz, M., Kowal, G., Otmianowska-Mazur, K., \& Lesch, H.: 2004, ApJL 605, L33

Hanasz, M., Kowalik, K., Wóltański, D., \& Pawłaszek, R.: 2008, ArXiv e-prints

Hanasz, M., Kowalik, K., Wóltański, D., \& Pawłaszek, R.: 2009a, ArXiv e-prints

Hanasz, M., Kowalik, K., Wóltański, D., \& Pawłaszek, R.: 2010a, in K. Goździewski, A. Niedzielski, \& J. Schneider (ed.), EAS Publications Series, Vol. 42 of EAS Publications Series, pp 275-280

Hanasz, M., Kowalik, K., Wóltański, D., Pawłaszek, R., \& Kornet, K.: 2010b, in K. Goździewski, A. Niedzielski, \& J. Schneider (ed.), EAS Publications Series, Vol. 42 of EAS Publications Series, pp 281-285

Hanasz, M. \& Lesch, H.: 2003, A\&SA 412, 331

Hanasz, M., Otmianowska-Mazur, K., Kowal, G., \& Lesch, H.: 2006, Astronomische Nachrichten 327, 469

Hanasz, M., Otmianowska-Mazur, K., Kowal, G., \& Lesch, H.: 2009b, A\&A 498, 335

Hanasz, M., Otmianowska-Mazur, K., \& Lesch, H.: 2002, A\&SA 386, 347

Hanasz, M., Wóltański, D., \& Kowalik, K.: 2009c, ApJL 706, L155

Jin, S. \& Xin, Z.: 1995, Comm. Pure Appl. Math. 48, 235

Kowal, G., Lazarian, A., Vishniac, E. T., \& Otmianowska-Mazur, K.: 2009, ApJ 700, 63

Kowal, G., Otmianowska-Mazur, K., \& Hanasz, M.: 2006, A $\&$ A 445, 915

Krause, M.: 2009, in Revista Mexicana de Astronomia y Astrofisica Conference Series, Vol. 36 of Revista Mexicana de Astronomia y Astrofisica, vol. 27, pp 25-29

Otmianowska-Mazur, K., Kowal, G., \& Hanasz, M.: 2007, ApJ 668, 110

Otmianowska-Mazur, K., Soida, M., Kulesza-Żydzik, B., Hanasz, M., \& Kowal, G.: 2009, ApJ 693, 1

Parker, E. N.: 1966, ApJ 145, 811

Parker, E. N.: 1992, ApJ 401, 137

Pen, U.-L., Arras, P., \& Wong, S.: 2003, ApJS 149, 447

Schlickeiser, R. \& Lerche, I.: 1985, A\&SA 151, 151

Siejkowski, H., Soida, M., Otmianowska-Mazur, K., Hanasz, M., \& Bomans, D. J.: 2010, A\&A 510, A97+

Wetzstein, M., Nelson, A. F., Naab, T., \& Burkert, A.: 2009, ApJS 184, 298

Wóltański, D., Hanasz, M., Kowalik, K., \& Kotarba, H.: 2011, ApJ(in prep) 